\title{
Permanent visual impairment in dengue fever following platelet transfusion: A series of 5 cases
}

\section{Dear Editor,}

Dengue fever (DF) is endemic in India and Singapore, and also causes frequent epidemics. Dengue virus belongs to the Flavivirus genus of the family Flaviviridae and its members include the 4 antigenically related serotypes of dengue virus (DENV 1-4). It is transmitted to humans through the bite of infected female Aedes aegypti mosquitoes, and is characterised by an acute onset of fever associated with symptoms of malaise, headache, muscle aches, retro-orbital pain, joint pain, abdominal discomfort, rash and bleeding diathesis.

Thrombocytopaenia, a major feature of DF, results from decreased platelet production from bone marrow suppression or increased platelet destruction. Prophylactic platelet transfusion is a common practice to prevent clinical bleeding in adults with dengue and thrombocytopaenia. However, transfusion of blood products may be detrimental to patients given the risks of fluid overload, transmission of infectious diseases and transfusion reactions.

Ocular findings associated with DF are subconjunctival haemorrhage, vitreous haemorrhage, retinal haemorrhage, cotton wool spots, central and branch retinal artery occlusion, central scotoma, papilloedema, optic neuropathy, retinal vasculitis, retinitis, retinal pigment epithelium mottling, foveolitis, choroidal effusion, exudative retinal detachment, anterior uveitis, endogenous endophthalmitis and panophthalmitis. ${ }^{1}$

Panophthalmitis is a rare complication, with only 7 cases reported following platelet transfusion in DF in the literature..$^{2-7}$ We report 5 cases of unusual complication of DF causing panophthalmitis, leading to rapidly progressive and painful visual loss shortly after receiving platelet transfusion. These 5 cases were referred from 5 different centres to our tertiary eye centre for ocular evaluation. As we are not aware of the prevailing practices in those centres, we are unable to comment on the rationale for platelet transfusion. These 5 cases occurred over a 6-month period from July to December 2019. Table 1 summarises these 5 cases.

Platelets are vulnerable to bacterial growth as they are stored at room temperature for up to 5 days, whereas other blood components are refrigerated or frozen. Gram-positive bacteria (e.g. Staphylococcus sp.) found on the skin are the most frequent contaminants of platelet units. Gram-negative bacteria (e.g. Serratia, Enterobacter or Salmonella sp.) account for more severe and fatal infections, and are attributed to donor bacteremia or contamination during product processing. ${ }^{8}$ Kuehnert et al. estimated that the rate of transfusion-transmitted bacteremia (in events/million units) was 9.98 for single-donor platelets, 10.64 for pooled platelets and 0.21 for red blood cell units. ${ }^{8}$ The US Food and Drug Administration requires only fatal complications of blood collection or transfusion to be reported. Antimicrobials and anti inflammatory agents in transfused populations may account for the partial masking of symptoms that are normally associated with sepsis. ${ }^{8}$

A study by Lee et al. on adult dengue patients in Singapore found that the occurrence of internal bleeding after platelet transfusion was slightly more common albeit statistically insignificant. ${ }^{9}$ More mucosal bleeding after platelet transfusion in transfused patients $(18.5 \%)$ versus non-transfused patients $(9.3 \%)$ was reported. In the transfused patients, platelet count increased significantly more the next day after platelet transfusion than in the non-transfused patients followed by a slow increase over the few days. Among the transfused patients, liver enzyme levels (aspartate aminotransferase and alanine aminotransferase) were found to be higher, 2 patients developed liver failure and 1 patient developed renal failure. ${ }^{9}$

Studies in India showed that acute respiratory distress syndrome, fever, longer duration of fever, longer duration of hospitalisation and slower recovery of platelets were more common in transfused patients compared to the control group. ${ }^{10}$

Approaches to reduce the incidence of transfusiontransmitted bacterial infection include expansion of donor screening, improved donor skin antisepsis, discarding an initial aliquot of donated blood to reduce skin contaminants, limitation of component storage time or lowering storage temperature, diagnostic screening of components and photochemical decontamination. $^{8}$

Frequency of blood component bacterial contamination associated with transfusion reaction (the BaCon Study) ${ }^{8}$ was initiated to better estimate their occurrence. Results showed no significant 


\begin{tabular}{|c|c|c|c|}
\hline 离 & 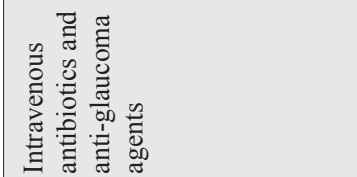 & 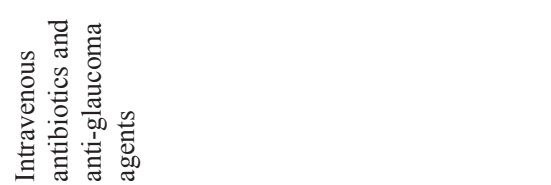 & 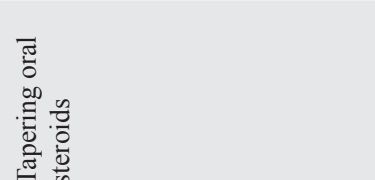 \\
\hline 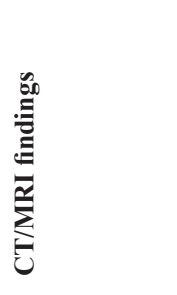 & 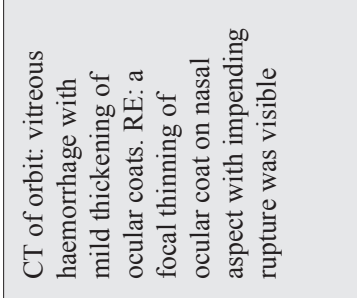 & 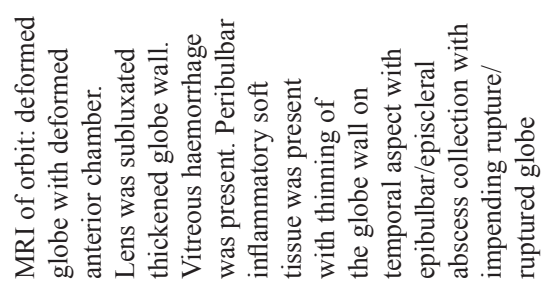 & 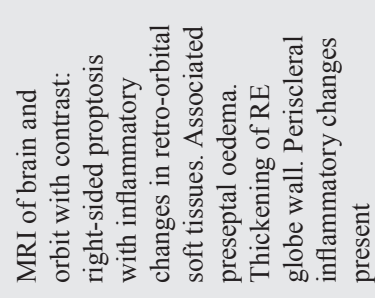 \\
\hline 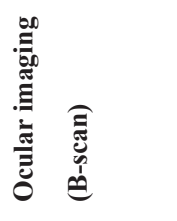 & 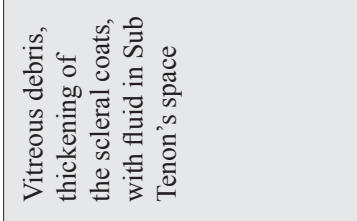 & 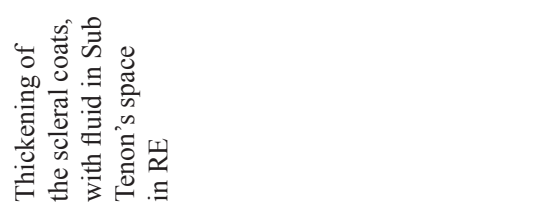 & 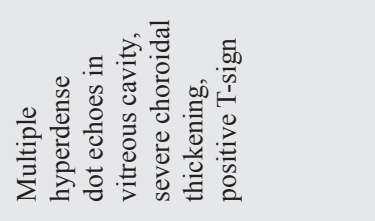 \\
\hline 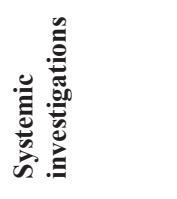 & 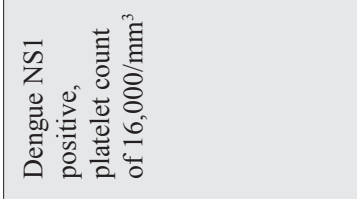 & 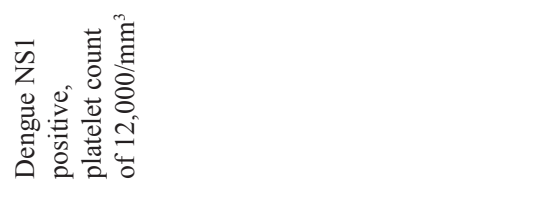 & 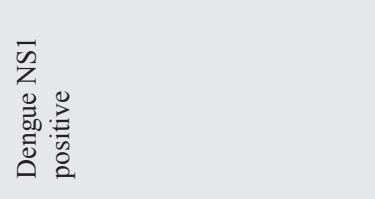 \\
\hline 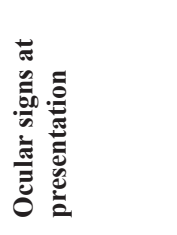 & 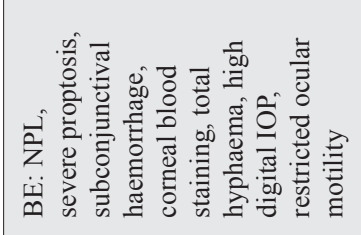 & 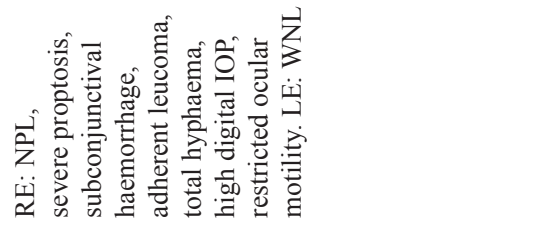 & 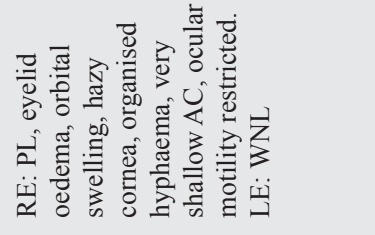 \\
\hline 产 & 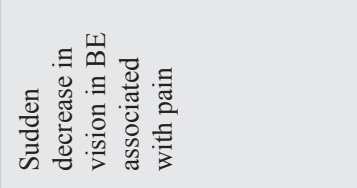 & 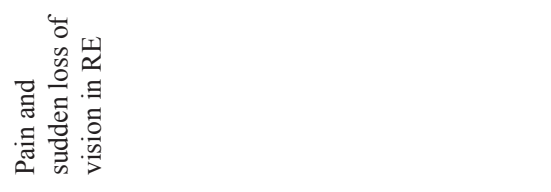 & 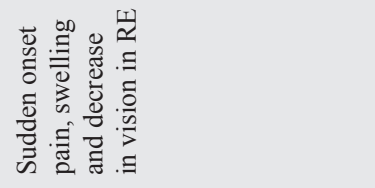 \\
\hline 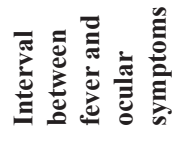 & 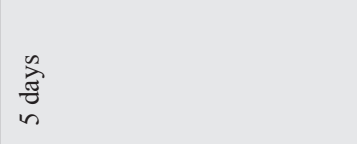 & $\begin{array}{l}\stackrel{0}{\vec{\theta}} \\
\stackrel{\sigma}{\sigma}\end{array}$ & 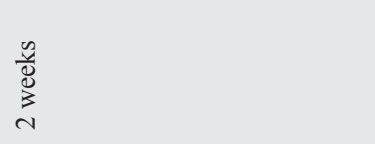 \\
\hline 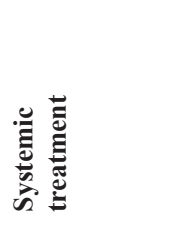 & 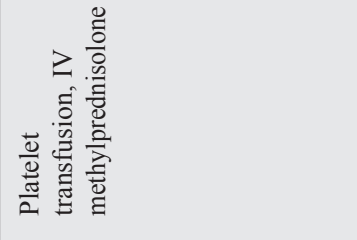 & 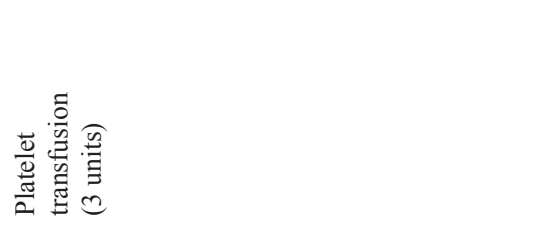 & 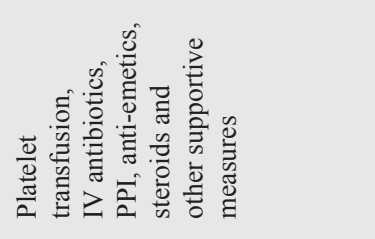 \\
\hline 总 & 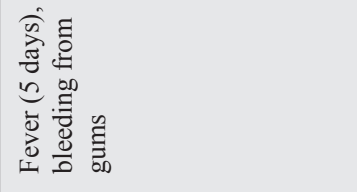 & 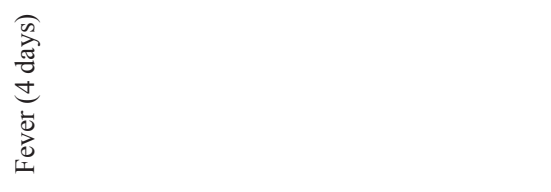 & 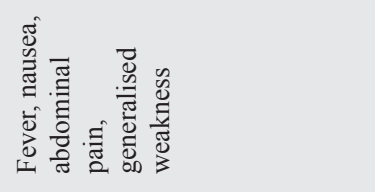 \\
\hline 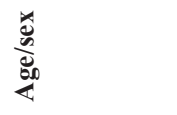 & $\frac{0}{\tilde{\pi}}$ & 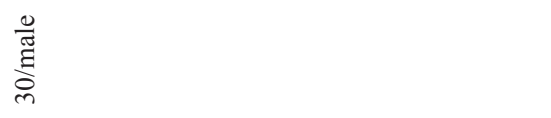 & 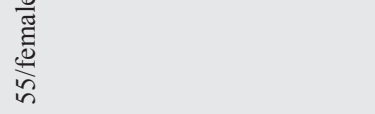 \\
\hline ن் & - & $N$ & $m$ \\
\hline
\end{tabular}




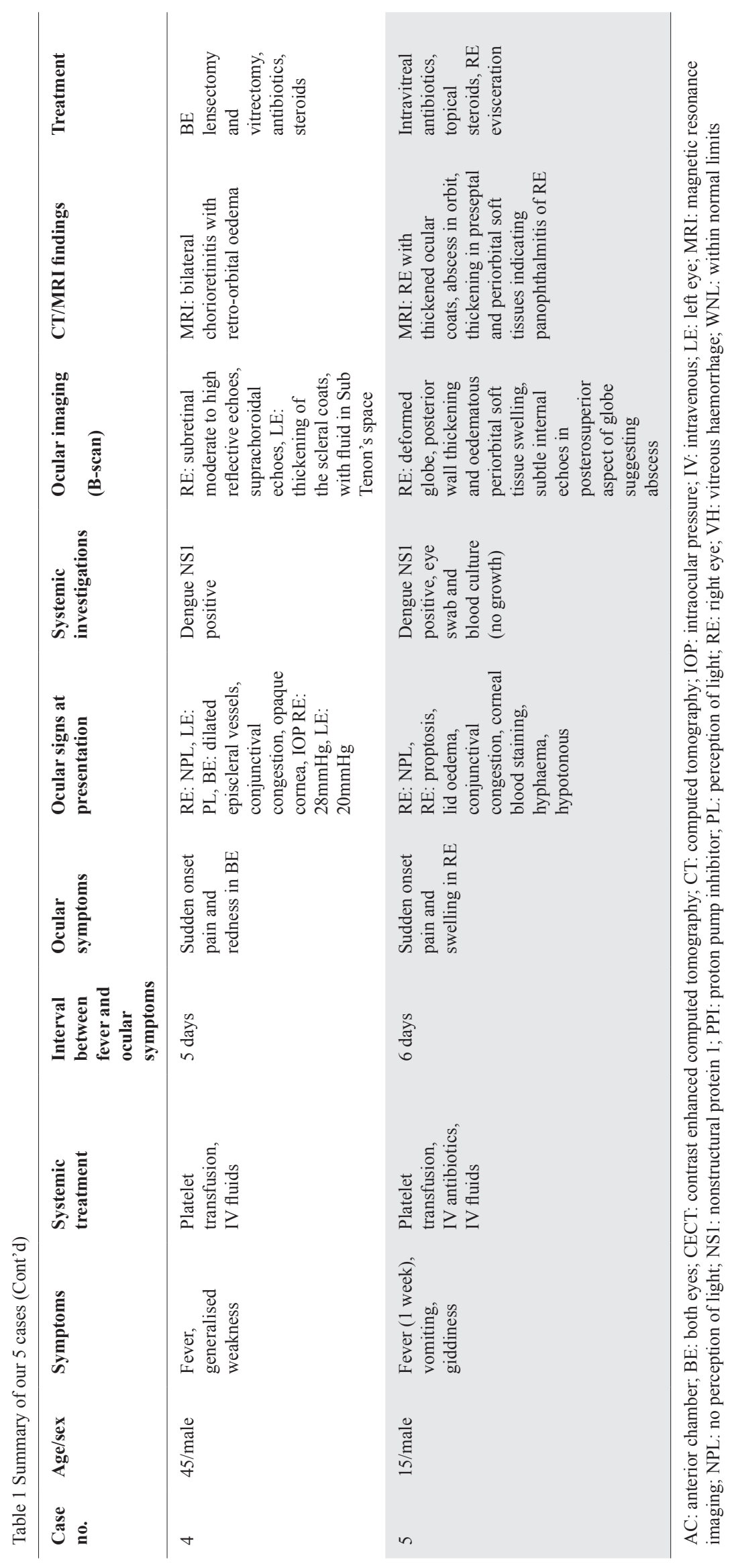


difference between the rates of transfusion-transmitted bacterial infection associated with single donor platelets and those associated with pooled platelets. ${ }^{11}$

Most DF-related ocular involvements are limited to the posterior segment, and manifest in the form of retinal vasculitis, retinitis, macular oedema or optic neuropathy. Endogenous endophthalmitis have been reported in DF and following platelet transfusion. ${ }^{5}$ Panophthalmitis associated with DF has been rarely reported. ${ }^{1}$

Panophthalmitis, a rare complication of DF, is an acute inflammation of all the coats of eyeball including intraocular structures. In normal circumstances, the blood ocular barrier provides a natural resistance against invading organisms. Panophthalmitis/ endophthalmitis may result in secondary to inflammatory or immune response to dengue virus; it may also lead to microorganisms crossing the blood ocular barrier to cause septic foci in the retina, which then spreads to the vitreous and anterior segments. Disintegration of the endothelial cells caused by antibodies against nonstructural protein 1 (NS1) antigen facilitates direct entry of the bacteria into the uveal and retinal circulation, causing septic foci and secondary endophthalmitis.

Five of the previous 7 published cases of dengue panophthalmitis had history of platelet transfusion. Five cases had unilateral involvement and 2 had bilateral involvement. The vision could not be salvaged in any of the affected eyes. Microorganisms isolated from the eye swab included gram-positive cocci with no growth, and Bacillus cereus was isolated from the eviscerated sample of the eye. ${ }^{4,5}$

In our series of 5 cases, the patients developed panophthalmitis shortly after receiving platelet transfusion and were referred to our tertiary eye centre. Average onset duration of complication was 5.5 days after transfusion (range of 4-7 days). Three cases had unilateral and 2 bilateral involvements respectively.

In 2 of our cases the presentation of panophthalmitis was initially atypical, hence an initial diagnosis of retrobulbar haemorrhage was made before referring to our centre. Both patients on ultrasound B scan had vitreous debris and thickening of the scleral coats, with fluid in Sub Tenon's space in the affected eyes and were blind. All the patients had undergone blood culture before referral to us with no reported growth of pathological organisms. At our tertiary eye centre, Gram's stain and potassium hydroxide mount and culture of the conjunctival swab did not show any microorganisms in all the 5 cases. Cases 4 and 5 underwent vitreous sampling and no growth of pathological organism was detected.

The crux in the management of dengue patients is maintaining good hydration, monitoring for any overt bleeding, alongside periodic checking on peripheral pulses and blood pressure with serial estimation of haematocrit and platelet counts. ${ }^{12}$ Supportive care consists of bed rest, fluid therapy and fever and pain relief medications.

The risk of transfusion-related adverse effects such as anaphylaxis, transmission of blood-borne infections and acute lung injury always exists with transfusion of blood products, including platelets. Prophylactic platelet transfusion has no added advantage over supportive care. The lack of efficacy of prophylactic platelet transfusion, combined with higher risk of adverse events, leads us to discourage the practice of routine prophylactic platelet transfusion in adult dengue.

Clinicians and ophthalmologists should be aware of this vision-threatening complication of DF for early recognition and prompt treatment. Vision could not be salvaged in any of the affected eyes in our 5 cases. Therefore, caution should be exercised while considering prophylactic platelet therapy in patients with DF.

Ophthalmological examination should be routinely done in all patients with DF. Patients with dengue who present with endophthalmitis or panophthalmitis should be aggressively managed, and samples (aqueous/ vitreous or eviscerated material) should be subjected to standard microbiological tests to investigate for offending organisms to tailor the therapy accordingly.

\section{REFERENCES}

1. Yip VC, Sanjay S, Koh YT. Ophthalmic complications of dengue fever: a systematic review. Ophthalmol Ther 2012;1:2.

2. Saranappa SBS, Sowbhagya HN. Panophthalmitis in dengue fever. Indian Pediatr 2012;49:760.

3. Sriram S, Kavalakatt JA, Pereira AL, et al. Bilateral panophthalmitis in dengue fever. Ann Trop Med Public Health 2015;8:217-8.

4. Kamal R, Shah D, Sharma S, et al. Culture-positive unilateral panophthalmitis in a serology-positive case of dengue hemorrhagic fever. Indian J Ophthalmol 2018;66:1017-9.

5. Ramananda K, Sundar MD, Mandal S, et al. Platelet Transfusion Related Panophthalmitis and Endophthalmitis in Patients with Dengue Hemorrhagic Fever. Am J Trop Med Hyg 2018;99:1053-4

6. Arya D, Das S, Shah G, et al. Panophthalmitis associated with scleral necrosis in dengue hemorrhagic fever. Indian J Ophthalmol 2019;67:1775-7

7. Kumar V, Deorari V, Swaroop S, et al. Panophthalmitis in a patient with dengue fever. BMJ Case Rep 2019; 12:e229588. 
8. Kuehnert MJ, Roth VR, Haley NR, et al. Transfusion-transmitted bacterial infection in the United States, 1998 through 2000. Transfusion 2001;41:1493-9.

9. Lee TH, Wong JG, Leo YS, et al. Potential Harm of Prophylactic Platelet Transfusion in Adult Dengue Patients. PLoS Negl Trop Dis 2016;10:e0004576.

10. Vishwanath M, Savitha MR, Krishnamurthy B. Platelet Transfusion in Dengue: Peril or Protection. Int J Med Res Rev 2015;3:303-7.

11. Roth VR, Kuehnert MJ, Haley NR, et al. Evaluation of a reporting system for bacterial contamination of blood components in the United States. Transfusion 2001;41:1486-92.

12. Kumar ND, Tomar V, Singh B, et al. Platelet transfusion practice during dengue fever epidemic. Indian J Pathol Microbiol 2000; 43:55-60.
Srinivasan Sanjay ${ }^{1}$ MRCS (Edin), Sameeksha Agrawal ${ }^{1}{ }_{M S(O p h)}$, Pooja Jain ${ }^{2} M S(O p h)$, Padmamalini Mahendradas ${ }^{1}{ }_{D N B}(O p h)$, Ankush $\underline{\text { Kawali }}{ }^{1}{ }_{D N B}(O p h)$, Naren $\underline{\text { Shetty }}{ }^{3} M S(O p h)$

1 Department of Uveitis and Ocular Immunology, Narayana Nethralaya, Bengaluru, India

2 Department of Cornea, Narayana Nethralaya, Bengaluru, India

3 Department of Cataract and Refractive Lens Services, Narayana Nethralaya, Bengaluru, India

Correspondence: Dr Srinivasan Sanjay, Department of Uveitis and Ocular Immunology, Narayana Nethralaya, 121/C Chord Road, Rajajinagar 1st R block, Bengaluru 560010, Karnataka, India.

Email: sanjaygroup24@gmail.com 